Europhys. Lett., 59 (2), pp. 311-317 (2002)

\title{
Kinetic pathway of spontaneous vesicle formation
}

\author{
J. Leng, S. U. EgelhaAf(*) and M. E. Cates \\ Department of Physics and Astronomy, The University of Edinburgh \\ Mayfield Road, Edinburgh EH9 3JZ, UK
}

(received 30 January 2002; accepted in final form 16 April 2002)

PACS. 82.70.Uv - Surfactants, micellar solutions, vesicles, lamellae, amphiphilic systems (hydrophilic and hydrophobic interactions).

PACS. 82.39.Rt - Reactions in complex biological systems.

PACS. 87.14.Cc - Lipids.

\begin{abstract}
The spontaneous formation of lipid vesicles (liposomes) in aqueous lecithin/bile salt mixtures is studied using time-resolved static and dynamic light scattering. These measurements reveal a strong dependence of the kinetic rates and end-state liposome properties on total amphiphile concentration and, even more pronounced, on ionic strength. The observed trends contradict equilibrium calculations, but are in quantitative agreement with a kinetic model that we present. This model identifies the key kinetic steps during vesicle formation: rapid formation of disc-like intermediate micelles, growth of these micelles and closure to form vesicles. This work offers conclusive evidence for kinetic rather than thermodynamic control of the end-state properties.
\end{abstract}

While the equilibrium behaviour of surfactant aggregates is well studied [1,2], their nonequilibrium behaviour has not been much addressed. Particularly interesting are transformations between different monolayer and bilayer topologies; a classical example is the micellevesicle transition [3,4]. It is, e.g., an important route to preparing lipid vesicles (liposomes) known as "reconstitution": a soluble detergent is removed from lipid-detergent micelles by dialysis or dilution, resulting in spontaneous vesicle formation $[4,5]$. This procedure is widely applied and, more generally, the properties of vesicles are extensively studied in a number of different systems. Nevertheless, there has been only limited understanding of their formation [5-11] and what determines their final ("end-state") properties; equilibrium calculations [12] give the wrong trends for the dependence of liposome size on bilayer composition [13-17]. This suggests a kinetic mechanism with the end-state vesicles being metastable structures that cannot achieve thermal equilibrium on observable timescales. The question whether vesicles represent equilibrium structures is, however, not unambiguously solved [13]. By combining light scattering experiments with a quantitative kinetic model (for full details see [18]), we conclusively establish a kinetic mechanism, at least for the systems we study, which comprise mixtures of lipid (lecithin) and natural detergent (bile salt).

$\left({ }^{*}\right)$ E-mail: S.U.Egelhaaf@ed.ac.uk

(C) EDP Sciences 

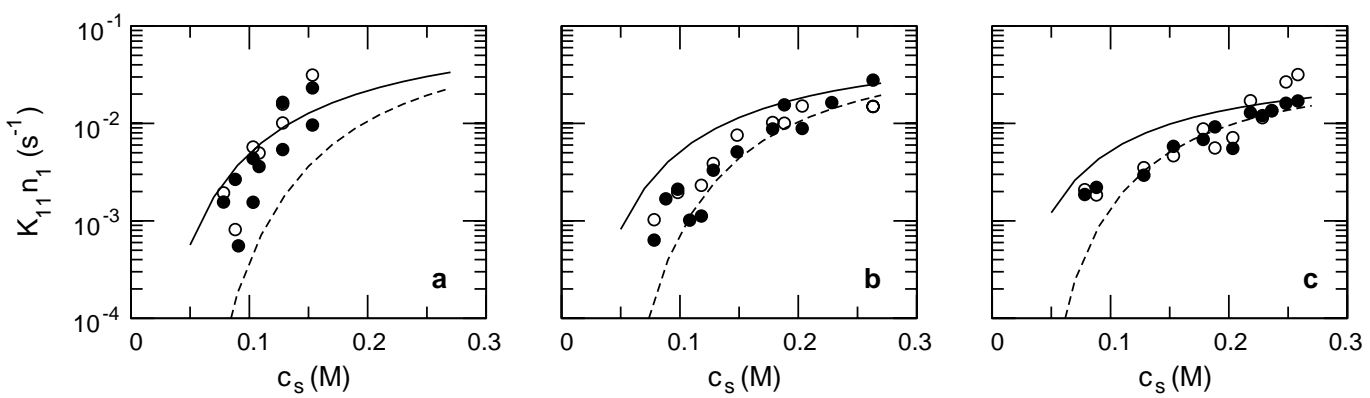

Fig. 1 - Rate of dimer formation $K_{11} n_{1}$ as a function of salt concentration $c_{\mathrm{s}}$ determined by static $(\bullet)$ and dynamic (o) light scattering. Final amphiphile concentrations are (a) $1.25 \mathrm{mg} / \mathrm{ml}$, (b) $0.83 \mathrm{mg} / \mathrm{ml}$ and (c) $0.50 \mathrm{mg} / \mathrm{ml}$. The electrostatic interactions between approaching disc-like micelles are calculated based on a constant potential (solid line) and constant charge (dashed line), respectively.

Aqueous lecithin-bile salt mixtures are prime examples of mixed amphiphile solutions that exhibit a spontaneous micelle-vesicle transition $[11,14-17,19]$. The average spontaneous curvature of a monolayer comprising lecithin and bile salt depends on its local composition: lecithin alone forms aggregates of low spontaneous curvature while bile salt alone forms highly curved (spherical) micelles. At high bile salt content thus spherical or elongated mixed micelles form. Since bile salt is far more soluble than lecithin, a subsequent dilution causes the composition of the aggregates to change, so that the spontaneous monolayer curvature decreases. With increasing dilution factor progressively longer cylindrical micelles are observed, until at higher dilution factors the end-state comprises near-monodisperse, unilamellar vesicles whose size decreases with dilution factor [14-17]. Time-resolved light and neutron scattering experiments suggest that on a sudden dilution spherical or elongated micelles very quickly change into disc-like micelles, which then transform into vesicles in a much slower process [19].

We have performed new time-resolved light scattering experiments to elucidate the pathway of vesicle formation and the role of kinetics in determining the end-state properties of the liposomes. Crucially, we not only varied the final total amphiphile concentration $c$, but also investigated the dependence on salt concentration $c_{\mathrm{s}}$. We expect $c_{\mathrm{s}}$ to control the electrostatic interactions between negatively charged bile salt molecules (mainly important at low $c_{\mathrm{s}}$ ) and to influence bile salt solubility, while having only marginal effects on the properties of the neutral lecithin within the range of $c_{\mathrm{s}}$ studied here [20].

The initial, equilibrated solutions of mixed micelles composed of lecithin (egg yolk lecithin; Nutfield Nurseries, GB) and bile salt (taurochenodeoxycholic acid sodium salt; Fluka) with a total amphiphile concentration of $25 \mathrm{mg} / \mathrm{ml}$ and a lecithin/bile salt molar ratio of 0.9 were prepared as previously described [17]. Solutions were centrifuged for $30 \mathrm{~min}$ at $5000 \mathrm{rpm}$ and $23{ }^{\circ} \mathrm{C}$ to remove dust and then rapidly diluted with carefully filtered buffer $(50 \mathrm{mM}$ Tris,

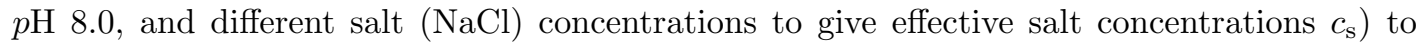
induce the micelle-vesicle transition. Vesicle formation was monitored by static and dynamic light scattering. The time dependencies of the average scattering intensity $I(t)$ and intensity correlation functions were monitored at a scattering angle of $90^{\circ}$ with a time resolution of $5 \mathrm{~s}$. Correlation functions were analysed using a second-order cumulant fit to obtain the average collective diffusion coefficient $D(t)$ from which the hydrodynamic radius $R_{\mathrm{h}}(t)$ is calculated.

The inital slopes of the relative scattered intensity $I(t) / I(0)$ and average collective diffusion coefficient $D(t)$ provide two independent methods to determine the initial rate of dimer formation $K_{11} n_{1}$, where $n_{1}$ is the number density of the initial disc-like micelles and $K_{11}$ the 

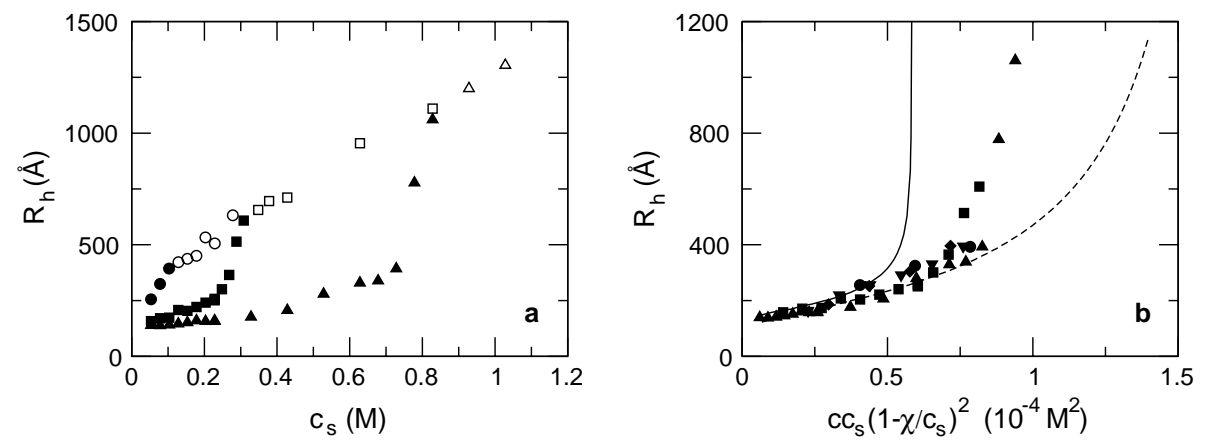

Fig. 2 - Hydrodynamic radius $R_{\mathrm{h}}\left(c, c_{\mathrm{s}}\right)$ measured in the end-state as a function of (a) salt concentration $c_{\mathrm{s}}$ and (b) rescaled concentration $c c_{\mathrm{s}}(1-\chi / c)^{2}$ for different final amphiphile concentrations $c$ $(1.25 \mathrm{mg} / \mathrm{ml}$ : circles, $1.00 \mathrm{mg} / \mathrm{ml}$ : diamonds, $0.83 \mathrm{mg} / \mathrm{ml}$ : inverted triangles, $0.63 \mathrm{mg} / \mathrm{ml}$ : squares, $0.42 \mathrm{mg} / \mathrm{ml}$ : triangles). Solid symbols correspond to vesicular samples and open symbols to samples in the micelle-vesicle coexistence region. Bounding predictions are shown as the dashed and solid lines (calculated for $c=0.42 \mathrm{mg} / \mathrm{ml}$ ).

reaction kernel for their coalescence into discs of twice the mass. We found both measurements to yield consistent values (fig. 1). On increasing $c_{\mathrm{s}}, K_{11} n_{1}$ shows a steep increase spanning about two decades, which is more pronounced for higher $c$. This concords with the increasing screening of Coulomb interactions at higher $c_{\mathrm{s}}$, and also with the presence of higher charge on the disc (i.e. higher bile salt content) at higher $c$.

In addition to these time-resolved experiments, the end-state samples were characterised using static and dynamic light scattering after the samples were left for at least two weeks at constant temperature $\left(T=23^{\circ} \mathrm{C}\right)$. We determined the hydrodynamic radius $R_{\mathrm{h}}$ (fig. $2 \mathrm{a}$ ), which in the case of vesicles is equal to the radius $R=R_{\mathrm{h}}$; the polydispersity, and the average aggregate mass, calculated from the scattering intensity extrapolated to zero scattering vector (data not shown). The general dependence of $R_{\mathrm{h}}$ on $c_{\mathrm{S}}$ is similar for all $c$ studied (fig. 2a). With increasing $c_{\mathrm{s}}$, first the mass and hydrodynamic radius $R_{\mathrm{h}}$ moderately increase before a pronounced growth is observed, whose onset, however, depends on $c$ (solid symbols). Then, a significant increase in polydispersity is noticed with a modest increase in the detected average hydrodynamic radius (open symbols); at the same time a dramatic drop in the average mass is observed. This indicates formation of another type of aggregate of lower scattering power, probably micelles, alongside the vesicles $[16,17]$. This is expected if the final bile salt concentration exceeds the maximum amount of bile salt which can be accommodated by vesicles (the "saturation concentration"). At even higher $c_{\mathrm{s}}$ (data not shown) the onset of bulk phase separation is detected, possibly to a lamellar phase.

Our model (fig. 3) assumes that the key kinetic steps are those between the rapidly formed, disc-like intermediate micelles and the end-state vesicles. We assume discs are made of (neutral) lecithin of negligible spontaneous curvature with (charged, fully dissociated) bile salt sequestered exclusively at the rim where the curvature is high. (We neglect the relatively small and roughly constant fraction of bile salt in the central part of the disc.) For our experimental conditions, the initial disc-like micelles are $d=50 \AA$ thick [19] and have a radius $r_{1}=60 \AA$ as determined from $D(t \rightarrow 0)$. The solubility of lecithin, about $10^{-10} \mathrm{M}$, is small enough to ignore molecular lecithin in solution. In contrast, bile salt has a higher solubility, about $10^{-3} \mathrm{M}$, and is thus partitioned between bulk solution (volume fraction $\phi_{\text {bulk }}$ ) and rims of discs (surface fraction $\phi_{\text {rim }}$ ). Immediately after dilution, conservation of total bile salt 


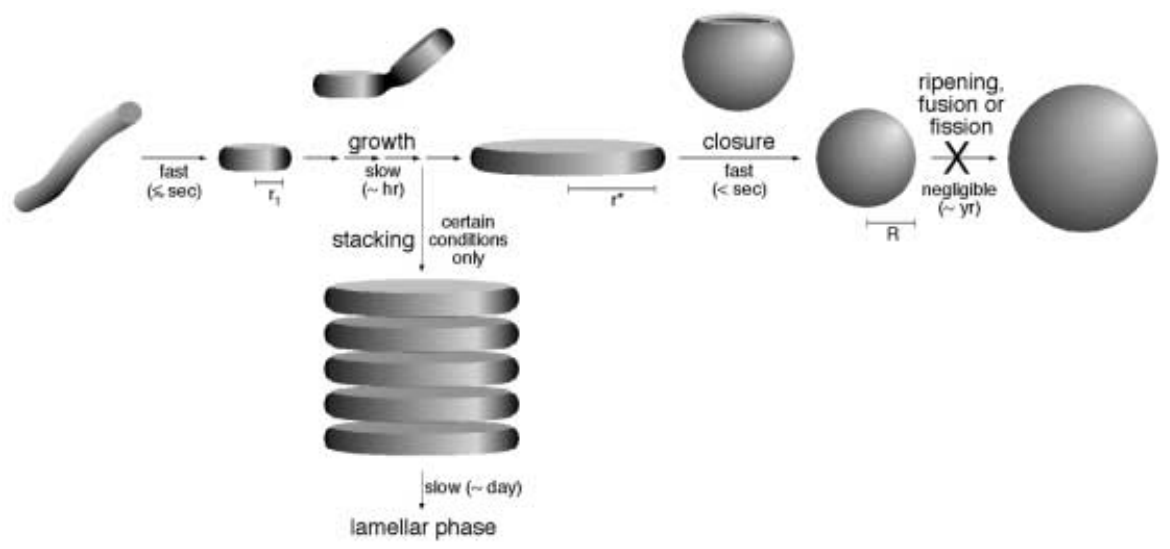

Fig. 3 - Schematic representation of our kinetic model of the micelle-vesicle transition. The fundamental steps and their typical time scales are shown: rapid formation of disc-like intermediate micelles, successive growth of these micelles up to their critical size (radius $r^{\star}$ ) followed by their closure to form vesicles. Ripening of these vesicles to their equilibrium size was not observed, but might occur on a very long time scale. Under certain conditions growth and closure becomes slower than stacking, which could lead to the formation of the lamellar phase. The transition states of coalescence, two discs joined by a "neck", and of closure, incomplete vesicle with a circular rim, are also indicated.

concentration $c_{\mathrm{BS}}$ requires

$$
c_{\mathrm{BS}}=\frac{\phi_{\mathrm{bulk}}}{N_{\mathrm{A}} v_{\mathrm{BS}}}+\frac{n_{1} S_{1} \phi_{\mathrm{rim}}}{N_{\mathrm{A}} a_{\mathrm{BS}}}
$$

where $S_{1}=\pi d^{2}\left(1+\pi r_{1} / d\right)$ is the area of the rim, $N_{\mathrm{A}}$ Avogadro's number, $v_{\mathrm{BS}}=660 \AA^{3}$ the volume of a bile salt molecule, and $a_{\mathrm{BS}}$ the rim area covered by a bile salt molecule at $\phi_{\text {rim }}=1$. The exchange of bile salt between bulk and rim is fast [19] and we assume local equilibrium for it. The area fraction of rim $\phi_{\text {rim }}$ covered by bile salt then depends on the bile

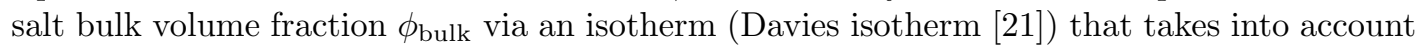
the micellization energy $\alpha_{\mathrm{m}}$ gained when one bile salt molecule is added to a disc-like micelle, as well as the electrostatic potential $\psi_{0}$ created by the charged bile salt molecules:

$$
\phi_{\text {rim }}=\frac{\phi_{\text {bulk }}}{\phi_{\text {bulk }}+\exp \left[-\left(\alpha_{\mathrm{m}}+e \psi_{0}\right) / k T\right]},
$$

where $e$ is the electronic charge and $k$ Boltzmann's constant. The electrostatic potential $\psi_{0}$ is related to the charge density on the rim and hence the bile salt surface density there, by [1]

$$
\phi_{\text {rim }}=-8 c_{\mathrm{S}} N_{\mathrm{A}} a_{\mathrm{BS}} \kappa_{\mathrm{D}}^{-1} \sinh \left(e \psi_{0} / 2 k T\right),
$$

where $\kappa_{\mathrm{D}}=\left(2 c_{\mathrm{S}} N_{\mathrm{A}} e^{2} / \epsilon k T\right)^{1 / 2}$ is the inverse Debye length and $\epsilon$ the dielectric constant of water. Equations (1)-(3) can be solved for $\phi_{\text {rim }}$, which depends on $c_{\mathrm{BS}}$ (and thus $c$ ) and $c_{\mathrm{S}}$. Under all conditions investigated $\phi_{\text {rim }}$ is significantly smaller than 1 , typically about 0.5 . This leads to local stress on the lecithin monolayer due to contact of hydrophobic parts with water, which causes an excess energy per unit length of rim, expressed as a line tension $\Lambda$ [22]:

$$
\Lambda=\Lambda_{0}\left[1+\frac{k T}{\alpha_{\mathrm{b}}} \ln \left(1-\phi_{\text {rim }}\right)\right]
$$


where $\Lambda_{0}$ is the line tension without detergent $\left(\phi_{\text {rim }}=0\right)$ and $\alpha_{\mathrm{b}}=2 \pi(r+d / 2) \Lambda_{0} a_{\mathrm{BS}} / S$ is the energy gain upon binding of one bile salt molecule to the rim. (We refer to a disc formed from $i$ initial disc-like micelles by subscript $i$, but for brevity suppress it when not referring to a specific size.)

We now assume a first stage in which disc-like micelles, initially monodisperse, grow by reaction-limited coalescence. (The low solubility of lecithin precludes growth by molecular diffusion or Ostwald ripening.) This is modelled using a set of Smoluchowski rate equations [23] whose kernel $K_{i j}=f_{i j} V_{i j} \Omega_{i j}$ describes coalescence of two discs, where $f_{i j} \propto\left(D_{i}+D_{j}\right) / \xi^{2}$ is an interaction-independent attempt frequency with $\xi$ the surface-to-surface separation of two discs at coalescence, $V_{i j} \approx 4 \pi r_{j}^{2}\left(1+r_{i} / r_{j}\right) \xi$ is an effective reaction volume (an integral over the configuration space in which coalescence can occur) and $\Omega_{i j}$ is a Boltzmann factor, which depends on the interaction potential. For the latter we take a standard DLVO form (including Deryaguin approximation) with, in addition, a local packing contribution to describe formation of a "neck" between two discs (fig. 3). This is smallest for rim-to-rim coalescence, outweighing the presence of a Coulomb barrier for this configuration. (This is evident from the strong $c_{\mathrm{s}^{-}}$ dependence of the initial rate $K_{11} n_{1}$ (fig. 1) which rules out face-to-face coalescence, given that the charged bile salt resides mainly on the rim.) To a good approximation, the DLVO contribution to the Bolztmann factor $\Omega_{\mathrm{e}, i j}$ can be separated from the packing part $\Omega_{\text {neck }}$, whose dependence on the discs sizes can be neglected, i.e. $\Omega_{i j} \approx \Omega_{\text {neck }} \Omega_{\mathrm{e}, i j}$, see ref. [18]. We can now in particular calculate $K_{11}$ based on which a characteristic growth time $\tau_{\mathrm{g}}$ for the coalescence of two initial discs can be defined as

$$
\tau_{\mathrm{g}}^{-1}=K_{11} n_{1} \approx f_{11} V_{11} \Omega_{\text {neck }} \Omega_{\mathrm{e}, 11} n_{1}=K_{11}^{0} n_{1} \Omega_{\mathrm{e}, 11} .
$$

$K_{11}^{0}$ comprises the dependence on the attempt frequency $f_{11}$ and on the neck packing energy together with a scale factor in the reaction volume (essentially $\xi$ ) and will be used as a fit parameter. (It only modifies the absolute value of $\tau_{\mathrm{g}}$, while the dependence on $c$ and $c_{\mathrm{s}}$ is contained in $n_{1}$ and $\Omega_{\mathrm{e}, 11}$.) Since the dependence of $K_{i j}$ on $i$ and $j$ is known, all $K_{i j}$ can be deduced as a function of $c$ and $c_{\mathrm{s}}$ once $K_{11}^{0}$ is fixed.

In a second stage, the larger disc-like micelles formed by coalescence close to form vesicles. This is driven by the line tension $\Lambda$, which will bend discs to decrease the length of the rim [22], and opposed by the bending modulus $\kappa$ and Gaussian modulus $\bar{\kappa}$ of the lecithin bilayer in the central part of the discs. The characteristic closure time $\tau_{\mathrm{c}}$ is estimated as

$$
\tau_{\mathrm{c}}=\tau_{\mathrm{z}} \exp \left[-E_{\mathrm{c}} / k T\right]=\tau_{\mathrm{z}} \exp \left[\frac{E_{\mathrm{s}}}{k T}\left(1-V_{\mathrm{f}} / 2\right)^{2}\right],
$$

with the inverse attempt frequency $\tau_{\mathrm{z}}=6 \pi \eta r^{3} / k T$ taken as a "Zimm time" of a disc of radius $r$ ( $\eta$ is the solvent viscosity), $E_{\mathrm{s}}=8 \pi(\kappa+\bar{\kappa} / 2)=8 \pi \tilde{\kappa}$ the elastic energy of a closed sphere and $V_{\mathrm{f}}=r \Lambda / 4 \tilde{\kappa}$ Fromherz's "vesiculation index" [22]. Growth of the disc-like micelles results in a drop of the barrier $E_{\mathrm{c}}$ which corresponds to the energy of a transition state made of an incomplete sphere with a circular rim (fig. 3). This leads to a very rapid decrease in closure time $\tau_{\mathrm{c}}$ beyond a certain disc radius, which depends on $c$ and $c_{\mathrm{s}}$. Equating $\tau_{\mathrm{c}}^{-1}(r)$ with the total coalescence rate of discs of size $r$ fixes the typical disc radius $r^{\star}$ at which closure occurs and thus sets the radius of the end-state vesicles $R=r^{\star} / 2$.

Two factors complicate a quantitative comparison between this kinetic model and experiment, cf. [18]. Firstly, calculation of $K_{i j}$ requires knowledge of how the bile salt on each of two approaching micellar rims is regulated in response to their growing Coulomb interaction. For this geometry the details are intractable, but we can calculate lower and upper bounds on the rates by assuming constant surface charge and constant surface potential, respectively. 
Secondly, as the disc-like micelles grow, the total length of rim present decreases with time. This leads to a redistribution of bile salt and steadily increasing $\phi_{\text {rim }}(t)$. Again, the details are intractable, but a lower bound on $\phi_{\text {rim }}(t)$ is that of the (actual) initial state, and an upper bound that of a (hypothetical) monodisperse set of discs of size $r^{\star}$. The first should be approached when the vesicle size $R$ is small; the second is more appropriate when $R$ is large.

To test our kinetic model, we now make a quantitative comparison between our calculations and measurements. The bilayer rigidity $\tilde{\kappa}=10 k T$, the solvent viscosity $\eta=10^{-3} \mathrm{Pas}$, and the Hamaker constant $A=5 \times 10^{-21} \mathrm{~J}$ (which enters the DLVO potential) are standard values; the four remaining parameters are fit globally to our kinetic and/or end-state data. The results for these parameters $\left(K_{11}^{0}=2 \times 10^{-23} \mathrm{~m}^{3} \mathrm{~s}^{-1}, \alpha_{\mathrm{m}}=10 k T, \Lambda_{0}=0.3 k T / \AA\right.$ and $a_{\mathrm{BS}}=200 \AA^{2}$ ) accord well with literature [24] and other estimates, such as the monomer solubility of bile salt. These values result in a characteristic growth time $\tau_{\mathrm{g}}$ of typically $100 \mathrm{~s}$ to $1000 \mathrm{~s}$. More importantly, the bounding estimates of $K_{11}$ can be compared to our kinetic data. Our model's predictions for constant charge (fig. 1, dashed lines) and constant potential (solid lines) are found to reproduce the main experimental trends on variation of $c$ and $c_{\mathrm{s}}$ and bracket the experimental data under most conditions. Likewise for the end-state vesicle size, our calculations based on the two limiting assumptions for $\phi_{\text {rim }}(t)$, initial conditions (fig. $2 \mathrm{~b}$, dashed line) and monodisperse growth (solid line), not only bracket the data, but show the expected trend from one limit to the other as the vesicle size evolves with $c$ and $c_{\mathrm{s}}$.

So far we focused our attention on the effect of $c_{\mathrm{s}}$; we now turn to the dependence on $c$, which leads to a pronounced shift of $R_{\mathrm{h}}\left(c_{\mathrm{s}}\right)$ (fig. 2a). Increasing $c$ also increases $\phi_{\text {rim }}$ (eq. (1)) and thus reduces $\Lambda$ (eq. (4)). It hence has a similar effect as increasing $c_{\mathrm{s}}$ (eqs. (3), (4)). This relation between $c$ and $c_{\mathrm{s}}$ can be quantified for a monodisperse population of growing large discs in the limit of small $\Lambda$. In this limit it is possible to derive, see [18], the scaling relationship $R_{\mathrm{h}}\left(c, c_{\mathrm{s}}\right)=f\left(c c_{\mathrm{s}}(1-\chi / c)^{2}\right)$, where $\chi=0.177 \mathrm{mg} / \mathrm{ml}$ based on the parameters given above. This scaling indeed leads to a collapse of the data (fig. 2b). This, alongside the other results of figs. 1 and 2, offers strong evidence for the kinetic model that we have described.

Our model can also be used to predict that under certain conditions further growth of the disc-like micelles and eventual closure becomes slower than stacking (fig. 3). This could correspond to the onset of phase separation to a lamellar phase [5,25]. This end-state could well be in equilibrium, but such a phase seems only to be reached if stacking of discs precludes their closure into (metastable) vesicles of indefinitely long lifetime.

The near-indefinite lifetime of the end-state liposomes, despite the fact that they are kinetic in origin, shows that both monomeric diffusion (Ostwald ripening) and vesicle fusion or fission are ineffective in bringing these liposomes to equilibrium. The first observation is consistent with the very low solubility of lecithin and the second with our finding that, among the disc-like intermediates, coalescence is rim-to-rim and not face-to-face. In contrast, relaxation to equilibrium may occur more rapidly for vesicles more prone to fusion or fission and/or formed by more soluble amphiphiles, such as some mixtures of anionic and cationic surfactants $[9,25,26]$. The ability to ripen or fuse thus plays an important role in determining whether vesicles reach equilibrium or represent kinetically trapped, metastable states of very long lifetime. This may give a new physicochemical basis for their classification [25]. (Note that a coarsening mechanism leading to partial equilibration could be mediated by mixed micelles in the coexistence region or simple bile salt micelles, if present.)

Our kinetic model identifies the important control parameters $\left(c, c_{\mathrm{s}}, \Lambda, \tilde{\kappa}, \alpha_{\mathrm{m}}\right)$ and fundamental steps during vesicle formation (rapid formation of disc-like intermediate micelles, growth of these micelles and closure to form vesicles; fig. 3) and highlights their influence on vesicle size. Although developed for aqueous mixtures of lecithin and bile salt, variants of 
the model could perhaps be applied to other amphiphile mixtures and/or amphiphile-protein mixtures which undergo a similar micelle-vesicle transition $[5,9,10,25-27]$.

In conclusion, the fact that our theoretical predictions reproduce the experimental trends indicates that the size of lecithin liposomes formed by a detergent removal technique (with bile-salt as the detergent) is controlled by kinetics, not thermodynamics. Particularly striking is the strong dependence of the end-state liposome size on $\mathrm{NaCl}$ concentration which, through its effect on the (charged) bile salt, modulates the kinetics although there is little bile salt left in the end-state vesicles (at least for the higher dilutions). The results explain why calculations based on thermodynamic equilibrium [12] predict a quite different dependence of liposome size than observed [13-17]. They also show that the kinetic pathways that arise under physiological conditions can sometimes best be studied by applying controlled deviations from these conditions.

We acknowledge support by EPSRC Grant GR/M89829 and Unilever plc.

\section{REFERENCES}

[1] IsRaelachvili J., Intermolecular and Surface Forces (Academic Press, London) 1992.

[2] Evans D. F. and Wennerström H., The Colloidal Domain - Where Physics, Chemistry, Biology, and Technology Meet (VCH Publishers, New York) 1994.

[3] Lasič D. D., Liposomes: From Physics to Applications (Elsevier Science, Amsterdam) 1993.

[4] Lichtenberg D., Handbook of Nonmedical Applications of Liposomes - Models for Biological Phenomena (CRC Press, Boca Raton) 1995, p. 199.

[5] Ollivon M., Lesieur S., Gabrielle-Mandelmont C. and Paternostre M., Biochim. Biophys. Acta, 1508 (2000) 34.

[6] Lasič D. D., J. Theor. Biol., 124 (1987) 35.

[7] Lasič D. D., Nature, 351 (1991) 613.

[8] Thompson T. E., Hepatology, 12 (1990) 51S.

[9] O'Connor A. J., Hatton T. A. and Bose A., Langmuir, 13 (1997) 6931.

[10] Campbell S. E., Zhang Z., Friberg S. E. and Patel R., Langmuir, 14 (1989) 590.

[11] Almog S., Kushnir T., Nir S. and Lichtenberg D., Biochemistry, 25 (1986) 2597.

[12] Safran S. A., Pincus P. and Andelmann D., Science, 248 (1990) 354.

[13] Kozlov M. M. and Andelmann D., Curr. Opin. Colloid Interface Sci., 1 (1996) 362.

[14] Schurtenberger P., Mazer N. and Känzig W., J. Phys. Chem., 87 (1983) 1042.

[15] Hjelm R. P., Alkan M. H. and Thiyagarajan P., Mol. Cryst. Liq. Cryst. A, 180 (1990) 155.

[16] Long M. A., Kaler E. W. and Lee S. P., Biophys. J., 67 (1994) 1733.

[17] Egelhajf S. U. and Schurtenberger P., J. Phys. Chem., 98 (1994) 8560.

[18] Leng J., Egelhata S. U. and Cates M. E., to be published.

[19] Egelhata S. U. and Schurtenberger P., Phys. Rev. Lett., 82 (1999) 2804.

[20] Meyuhas D., Bor A., Pinchuk I., Kaplun A., Talmon Y., Kozlov M. M. and LichtenBERG D., J. Colloid Interface Sci., 188 (1997) 351.

[21] Davies J. T., Proc. R. Soc. London, Ser. A, 245 (1958) 417.

[22] Fromherz P., Chem. Phys. Lett., 94 (1983) 259.

[23] Hänggi P., Talkner P. and Borkovec M., Rev. Mod. Phys., 62 (1990) 251.

[24] Moroz J. D. and Nelson P., Biophys. J., 72 (1997) 2211.

[25] Marques E. F., Langmuir, 16 (2000) 4798.

[26] Kaler E. W., Murthy A. K., Rodriguez B. E. and Zasadzinski J. A. N., Science, 245 (1989) 1371.

[27] Almgren M., Biochim. Biophys. Acta, 1508 (2000) 146. 